\title{
CONFLICT, AROUSAL, AND LOGICAL GUT FEELINGS
}

\author{
Wim De Neys $s^{1,2,3}$
}

1 - CNRS, Unité 3521 LaPsyDÉ, France

2 - Université Paris Descartes, Sorbonne Paris Cité, Unité 3521 LaPsyDÉ, France

3 - Université de Caen Basse-Normandie, Unité 3521 LaPsyDÉ, France

Mailing address: Wim De Neys

LaPsyDÉ (Unité CNRS 3521, Université Paris Descartes)

Sorbonne - Labo A. Binet

46, rue Saint Jacques

75005 Paris

France

wim.de-neys@parisdescartes.fr 


\begin{abstract}
Although human reasoning is often biased by intuitive heuristics, recent studies on conflict detection during thinking suggest that adult reasoners detect the biased nature of their judgments. Despite their illogical response, adults seem to demonstrate a remarkable sensitivity to possible conflict between their heuristic judgment and logical or probabilistic norms. In this chapter I review the core findings and try to clarify why it makes sense to conceive this logical sensitivity as an intuitive gut feeling.
\end{abstract}




\section{CONFLICT, AROUSAL, AND LOGICAL GUT FEELINGS}

Imagine you're on a game show. The host shows you two metal boxes that are both filled with $\$ 100$ and $\$ 1$ dollar bills. You get to draw one note out of one of the boxes. Whatever note you draw is yours to keep. The host tells you that box A contains a total of 10 bills, one of which is a $\$ 100$ note. He also informs you that Box B contains 1000 bills and 99 of these are $\$ 100$ notes. So box $\mathrm{A}$ has got one $\$ 100$ bill in it while there are 99 of them hiding in box $\mathrm{B}$. Which one of the boxes should you draw from to maximize your chances of winning $\$ 100$ ?

When presented with this problem a lot of people seem to have a strong intuitive preference for Box B. From a logical point of view, this is not a smart choice. Indeed, although Box $B$ contains far more $\$ 100$ bills than box $A$, there are also a lot more $\$ 1$ notes in box $B$. If you take the ratio of $\$ 1$ and $\$ 100$ bills in both boxes into account it is clear that Box $A$ is giving you a $10 \%$ chance of picking a $\$ 100$ bill (i.e., $1 / 10$ ) while Box B only offers a $9.9 \%$ chance (i.e., 99/1000). The striking thing is that although one doesn't need to be a math genius or logician to figure this out, many educated people nevertheless fail to solve this basic "ratio" problem (Epstein, 1994). The fact that the absolute number of $\$ 100$ bills is so much higher in Box B has such a strong intuitive pull that they seem to neglect the ratio principle and erroneously conclude that they should draw from box B.

Decades of reasoning and decision-making research have shown that similar intuitive thinking is biasing people's judgment in a wide range of situations and tasks (Evans, 2008; Evans \& Over, 1996; Kahneman \& Frederick, 2005; Kahneman \& Tversky, 1973). In general, human reasoners seem to have a strong tendency to base their judgment on fast intuitive impressions 
rather than on more demanding, deliberative reasoning. Although this intuitive or so-called "heuristic" thinking might sometimes be useful, it will often cue responses that conflict with more logical or probabilistic principles and bias our reasoning (Evans, 2003, 2010; Kahneman, 2011; Stanovich \& West, 2000).

If you failed to solve the introductory problem you might find some comfort in the fact that you're far from being the only one who gets fooled when solving these problems. Moreover, even if you fell trap to the intuitive bias you might have been less ignorant than what your erroneous response suggests. Indeed, you might have picked the incorrect response, but were you actually fully convinced that your answer was right? That is, the problem might have tempted you to pick the heuristic response, but you might have felt that there was something tricky about the problem and that you were missing out on something. In this chapter I will be writing about that very feeling. Recent studies on conflict detection during thinking show that despite the omnipresent bias, people are remarkably sensitive to violations of logical and probabilistic principles when they reason (e.g., Bonner \& Newell, 2010; De Neys \& Franssens, 2009; De Neys \& Glumicic, 2008; Stupple \& Ball, 2008). Bluntly put, although people are often biased and fail to give the correct response they also seem to implicitly detect that they are erring.

I have organized the chapter around brief sections. I will start by sketching the basic rationale and core findings of the conflict detection studies. Next, I'll try to clarify why it makes sense to conceive people's logical sensitivity as an intuitive gut feeling. Finally, l'll comment on the possible affective basis of the experienced conflict and the possible link between logical gut feelings and Damasio's (1994) somatic marker hypothesis. 
I should stress that I have tried to write the present chapter with a non-reasoning expert audience in mind. The expert reader might want to note that a more detailed version of the basic idea that I am presenting here can be found elsewhere (see De Neys, 2012 and De Neys, Moyens, \& Vansteenwegen, 2010) ${ }^{1}$.

\section{CONFLICT DETECTION STUDIES}

Research on conflict detection during thinking has focused on people's processing of the infamous classic reasoning tasks that have been studied for decades in the reasoning and decision making field (e.g., base-rate neglect tasks, ratio-bias tasks, conjunction fallacy, belief bias syllogisms, etc.). Giving the correct response in these tasks requires only the application of some very basic logical or probabilistic principles. However, as the introductory ratio-bias

\footnotetext{
${ }^{1}$ For completeness, I should also warn the hardcore reasoning psychologist - especially if they are a bit fetishist about nomenclature - that I will be using the label "correct" or "logical" response as a handy shortcut to refer to "the response that has traditionally been considered as correct or normative according to standard logic or probability theory". The appropriateness of these traditional norms has sometimes been questioned in the reasoning field (e.g., see Stanovich \& West, 2000, for a review). Under this interpretation, the heuristic response should not be labeled as "incorrect" or "biased". For the sake of simplicity I stick to the traditional labeling here. In the same vein, I use the term "logical" as a general header to refer both to standard logic and probability theory.
} 
example illustrated, the tasks are constructed such that they intuitively cue a tempting heuristic response that conflicts with these principles. The basic question that the detection studies have been trying to answer is whether people are sensitive to this conflict and notice that their heuristic response is questionable. To do this the studies typically contrast people's processing of the classic problems with newly constructed control versions. In the control or no-conflict versions the conflict is removed and the cued heuristic response is consistent with the logical response. For example, a no-conflict control version of the introductory $\$ 100$ bill problem could simply state that box A contains 20 (instead of 10) bills. Everything else stays the same. In this case both the absolute number of $\$ 100$ bills (i.e., 1 vs. 99) and the ratio of $\$ 100 / \$ 1$ bills (i.e., 1/19 vs. 99/901) would be higher in box B. Hence, both heuristic considerations based on the absolute number and logical ratio considerations cue the exact same response.

The conflict detection studies have introduced a wide range of subtle processing measures to examine whether people process the conflict and no-conflict versions differently. For example, one basic procedure has been to simply look at people's response latencies: A number of studies reported that people need typically more time to solve the conflict than the control versions (e.g., Bonner \& Newell, 2010; De Neys \& Glumicic, 2008; Stupple \& Ball, 2008; Thompson, Striemer, Reikoff, Gunter, \& Campbell, 2003; Villejoubert, 2009; but see also Pennycook, Fugelsang, Koehler, 2012). Now, the only difference between the two versions is whether the cued heuristic response is consistent with the correct logical response or not. For example, in the $\$ 100 / \$ 1$ bill problem the only modified factor in the control version would be the ratio of bills in box A. If biased reasoners were really mere heuristic thinkers who only focused on the absolute number of $\$ 100$ bills, they should not process the two types of 
problems any differently. Hence, the latency findings support the idea that people are sensitive to the logical status of their judgment: If people's intuitive heuristic answer conflicts with the logical norm, their problem processing time will increase.

Further support for this claim has come from gaze and eye-tracking studies that showed that the longer latencies are specifically accompanied by a longer inspection of logically critical problem information (e.g., Ball, Philips, Wade, \& Quayle, 2006; De Neys \& Glumicic, 2008). For example, in one study Ball et al. (2006) recorded participants' eye-movements while they were solving conflict and no-conflict syllogisms. In a conflict syllogism the believability of the conclusion conflicts with its logical validity (e.g., a problem with an invalid but believable conclusion). Consider the following example:

Premisses: All fruit can be eaten. Apples can be eaten.

Conclusion: Apples are fruit.

Is the conclusion logically valid?

a. yes

b. no

Intuitively, participants will be tempted to judge the validity by evaluating its believability. Consequently, people frequently err when solving the conflict versions. However, in the noconflict version the believability of the conclusion will be consistent with its logical status (e.g., a believable and valid conclusion). Consider the following example:

Premisses: All fruit can be eaten.

Apples are fruit.

Conclusion: Apples can be eaten. 
Is the conclusion logically valid?

a. yes

b. no

Ball et al. (2006) observed that after participants read the conclusion of a conflict syllogism they made saccades to the premises and started re-inspecting this information. Such "reviewing" was found to be much less pronounced on the no-conflict versions. Hence, despite the frequent logical errors on the conflict problems, people did seem to show some basic sensitivity to the belief-logic conflict.

A similar gaze trend has been observed with base-rate problems (e.g., De Neys \& Glumicic, 2008). In these problems a stereotypical personality description cues a heuristic response that can conflict with logically critical base-rate information. Consider the following example:

A psychologist wrote thumbnail descriptions of a sample of 1000 participants consisting of 5 Italians and 995 Swedish people. The description below was drawn randomly from the 1000 available descriptions.

Mario is 25 years old. He is a charming young man and is a real womanizer. His favorite dish is the spaghetti his mother makes.

What is most likely?

a. Mario is Italian.

b. Mario is Swedish.

Intuitively, many people will be tempted to conclude that Mario is Italian based on stereotypical beliefs cued by the description. However, given that there are far more Swedes than Italians in the sample (i.e., 995 out of 1000) the statistical base-rates favor the conclusion that a randomly drawn individual will most likely be a Swede. Hence, logically speaking, taking the base-rate into 
account should push the scale to the "Swede" side. Note that in the no-conflict version of this problem the base-rates would simply be switched around (e.g., a sample of 995 Italians and 5 Swedes) so that both the base-rates and description cue the same response. In line with Ball et al.'s (2006) syllogistic reasoning findings, De Neys and Glumicic observed that although participants typically failed to give the correct base-rate response in the classic conflict version, they nevertheless showed an increased tendency to re-view the paragraph with the base-rate information after they read the personality description.

Interestingly, the behavioral conflict findings have been validated with a brain-based approach. De Neys, Vartanian, and Goel (2008) used fMRI to monitor the activation of a specific brain area, the anterior cingulate cortex (ACC), which is believed to mediate conflict detection during thinking (e.g., Botvinick, Cohen, \& Carter, 2004). Participants were given classic conflict base-rate problems and the no-conflict control versions. In line with the behavioral findings, results showed that the ACC was much more activated when people solved the conflict versions than when they solved the control versions. In a subsequent study, participants' skinconductance was recorded to monitor autonomic nervous system activation while solving conflict and no-conflict syllogisms (De Neys et al., 2010). Results showed that solving the conflict problems resulted in a clear electrodermal activation boost. Hence, in addition to the ACC activation, solving conflict problems literally aroused participants. These neural conflict signals have also been shown to affect people's subjective response confidence: Biased participants typically indicate that they feel less confident about their answer after solving conflict problems than after solving the control problems (e.g., De Neys, Cromheeke, \& Osman, 2011). 
In sum, the conflict detection studies indicate that although people might be often biased and fail to give the correct logical answer on many reasoning tasks, they are not completely oblivious to their bias. Reasoners show some basic sensitivity to the fact that their heuristic answer conflicts with logical considerations. This conflict sensitivity entails that they do not simply disregard the logical implication of their judgments.

\section{LOGICAL GUT FEELINGS}

Establishing that people might show some logical sensitivity is one thing. However, a critical open question is how this sensitivity needs to be conceived. To detect conflict between intuitively cued heuristic intuitions and logical considerations, this logical knowledge needs to be activated at some level. I have argued (De Neys, 2012) that that this knowledge is intuitive in nature and is activated automatically when people are faced with a reasoning task - hence, the idea of a logical intuition. In other words, I suggest that in addition to the well established heuristic response, the classic tasks also automatically evoke an intuitive logical response. Whenever these responses conflict, this will create arousal. People will notice this arousal and this results in a questioning of the heuristic response. However, people will typically not manage to label this experience explicitly - hence, the idea of a logical "gut feeling". I discuss some basic evidence for this characterization below.

In one of the first experiments that I ran on conflict detection, Tamara Glumicic and I decided to adopt a thinking-aloud procedure to examine people's explicit conflict sensitivity (De Neys \& Glumicic, 2008). We presented participants with base-rate neglect problems such as the 
Italians and Swedes problem that I introduced earlier and asked them to say out loud what they were thinking about when they tried to solve the problem. We reasoned that if participants explicitly detected the conflict between the cued heuristic response and the base-rate information, they would at the very least refer to the base-rate information. Hence, we figured that people would say something like "I think this guy will be an Italian because he likes spaghetti ... but there are more Swedes too ...". However, results of two independent studies we ran were strikingly clear: people hardly ever explicitly referred to the base-rate information when solving the classic conflict versions (see De Neys and Glumicic, 2008). Hence, although we later established that participants needed more time to solve these problems, made eyemovements to the base-rate information, showed increased ACC activation, increased autonomic arousal, and decreased response confidence when solving these very same problems, they did not verbally express that the base-rates mattered. Hence, at the explicit, verbal level there seems to be little detection or logical sensitivity going on. In general, this fits with the long established observation that people's online verbalizations during thinking and their retrospective response justifications do typically not indicate that they are taking logical or probabilistic considerations into account (e.g., Evans \& Over, 1996; Wason \& Evans, 1975). In sum, it is quite clear that people will not manage to label explicitly the logical violations that they do seem to be detecting. For me this was one of the reasons to claim that the logical conflict sensitivity we demonstrated was implicit and should be conceived as a "gut feeling": People will be aware that there is something fishy about their heuristic response, but they will not be able to put their finger on it and explain why their response is questionable. More precisely, as indicated above, the idea that I propose is that the conflict between intuitively 
activated logical principles and the cued heuristic response creates arousal. People experience this arousal, this makes them doubt their heuristic response, but they will not be able to justify why their response is questionable. However, the implicit knowledge - or the logical intuition as I like to call it - suffices to signal that the heuristic response is not fully warranted.

Further evidence for the implicit or intuitive nature of people's logical sensitivity comes from the apparent automaticity of the process. Detecting conflict has been shown to be quite effortless. For example, people's conflict sensitivity is not hampered when their cognitive resources are burdened by a secondary task (e.g., Franssens \& De Neys, 2009). In addition, individual differences in cognitive capacity seem to have little impact on people's conflict sensitivity. The detection studies that I reviewed above clearly established that even the least gifted reasoners (i.e., the most biased reasoners with the lowest accuracy scores - who typically have the lowest scores on cognitive capacity tests, e.g., see Stanovich \& West, 2000 -) showed the reported conflict sensitivity effects (e.g., De Neys \& Glumicic, 2008; De Neys et al., 2010, 2011).

Finally, I believe that the intuitive character of the postulated logical sensitivity is also supported by the fascinating recent work of Morsanyi and Handley (2012). These authors presented participants with syllogistic reasoning problems and simply asked them whether they liked the conclusion or not. In line with the conflict detection findings, they observed that although people typically failed to solve these problems correctly, the participants did like invalid problems (i.e., problems with a logically erroneous conclusion) far less than valid problems (i.e., problems with a logically correct conclusion, but see also Klauer \& Singmann, 2012 ). Interestingly, in one condition they adopted a so-called misattribution paradigm (e.g., 
Topolinski \& Strack, 2009) and asked people to rate the likability while they were listening to classical music - a fragment from Claude Debussy's "La Mer" - that was played in the background. Participants were led to believe that previous research had shown that the music would influence their emotional reactions. Morsanyi and Handley observed that under these instructions the likability rating no longer differed for valid and invalid problems. Consistent with the present suggestion, Morsanyi and Handley argued that since people would not be aware of the source of their intuitive logical judgment, they would misattribute the experienced arousal to the music, and thereby eliminate the effect on their liking judgment.

\section{AFFECTIVE CONFLICT?}

To recap, the idea that I propose is that the conflict between intuitively activated logical principles and a cued heuristic response creates arousal. People experience this arousal and this suffices to signal that the heuristic response is not fully warranted. Thompson and Morsanyi (2012) recently suggested that the experienced arousal might have an affective basis that would ultimately be grounded in the fluency with which an answer is produced. In terms of this model, conflict between a heuristic intuition and implicit logical knowledge would result in disfluent processing (e.g., Alter, Oppenheimer, Epley, \& Eyre, 2007; Oppenheimer, 2008). This in turn creates negative affect that results in a questioning of the heuristic response (or a low "Feeling of Rightness" as Thompson, 2009, puts it; see also Thompson, Turner, \& Pennycook, 2011). The interested reader might note that related support for a potential affective basis can be found in Topolinski's (2011) Fluency-Affect-Intuition model (2011). 


\section{LOGICAL GUT FEELINGS AND SOMATIC MARKERS}

The claim that people intuitively detect their reasoning bias might make some readers wonder about a possible link between the conflict detection findings and the seminal work of Damasio and colleagues (e.g., Bechara, Damasio, Tranel, \& Damasio, 1997; Damasio, 1994). In Damasio's studies, participants were presented with a gambling task in which they could select cards from decks with different payoffs. Damasio and colleagues observed that participants needed about 80 trials before they could explicitly point out which decks were the good and bad ones. However, when the authors examined people's autonomic responses they noticed that after a few trials (i.e., between the 10th and 50th trial) people started showing a skin conductance increase (i.e., a "somatic marker") before they selected a card from the bad decks. Hence, although people had not yet acquired explicit knowledge of which decks were the bad ones, their autonomic response indicated that they implicitly acquired this knowledge.

It will be evident that both Damasio's work and the conflict detection work that I presented here share the general idea that there is "more than meets the eye" to human thinking: Although people's overt response (i.e., their answer on the reasoning tasks) might be biased, this does not imply that they cannot detect this bias at a more implicit level. However, at the same time I would like to point out that the autonomic signal that Damasio focused on is different from the conflict driven autonomic arousal signal that I am talking about here. First, Damasio and colleagues argued that the autonomic reaction in their gambling task resulted from the negative feedback (i.e., losing money) participants received after selecting bad cards. 
In the classic bias tasks that were studied in the conflict detection studies such performance feedback was completely absent. Furthermore, item analyses show that the conflict sensitivity effects are present from the first problem presentation and do not result from a learning effect after solving multiple trials (e.g., De Neys \& Franssens, 2009; De Neys \& Glumicic, 2008; De Neys et al., 2010, 2011). In addition, Damasio only observed his somatic markers before participants made erroneous selections. As one would expect from a conflict related detection signal, the increased arousal in the conflict detection studies was observed both for biased and correct answers (e.g., De Neys et al., 2008, 2010, 2011). Lastly, somatic markers and conflict feelings might also have different neural underpinnings. Note that the somatic markers have been shown to be generated by the ventromedial prefrontal cortex (vMPFC, e.g., Bechara et al., 1997). As I reviewed above, available evidence suggests that the critical brain structure for the logical conflict signal is the more dorsal anterior cingulate cortex (ACC, e.g., De Neys et al., 2008). Interestingly, the VMPFC and ACC have been associated with two distinct functionalanatomical networks within the prefrontal cortex (Gläscher et al., 2012). According to this research the VMPFC would be part of a more "motivational" network associated with valuebased decision making whereas the ACC would be part of a "control" network associated with cognitive control and conflict monitoring ${ }^{2}$.

\footnotetext{
${ }^{2}$ To avoid confusion, note that this point does not allude to the old-school distinction between "cognitive" and "emotional" brain networks. Research on cognitive control suggests that even the ACC-centered control network can have an affective basis (Proulx, Inzlicht, Harmon-Jones, 2012 ). In addition, as I clarified in the previous section, there are good independent reasons to assume that the conflict signal during thinking is indeed affective in nature. The claim is simply
} 
In sum, although both the conflict findings and Damasio's work point to the importance of the autonomic response level in human thinking, the two bodies of work seem to deal with different autonomic signals. Obviously, it will be interesting to examine the precise relation between the two signals in future studies.

\section{IN CLOSING}

In my work I have been trying to convince the reasoning and decision-making community that the idea that people have logical gut feelings is valuable and should become a primary area of future empirical and theoretical scrutinizing. As I mentioned in the introduction, my primary goal in this chapter was to sketch an accessible picture of the basic findings and claims for a non-reasoning expert audience. I specifically hope that this chapter (and book) might help to attract more interest from people working in the emotion field. My gut feeling is telling me that the methods and expertise of emotion researchers will be extremely helpful to further pinpoint the nature of the logical gut feelings and the precise mechanism by which they come to affect our judgment.

\section{ACKNOWLEDGEMENTS}

that logical gut feelings and somatic markers are presumably generated by different brain networks. 
Preparation of this manuscript was supported by a grant from the Agence National de la Recherche (ANR-12-xxxx-000).

\section{REFERENCES}

Alter, A. L., Oppenheimer, D. M., Epley, N., \& Eyre, R. N. (2007). Overcoming intuition: metacognitive difficulty activates analytic reasoning. Journal of Experimental Psychology: General, 136, 569-576.

Ball, L. J., Philips, P., Wade, C. N., \& Quayle, J. D. (2006). Effects of belief and logic on syllogistic reasoning: Eye-movement evidence for selective processing models. Experimental Psychology, 53, 77-86.

Bechara, A., Damasio, H., Tranel, D., \& Damasio, A. R. (1997). Deciding advantageously before knowing the advantageous strategy. Science, 275, 1293-1295.

Bonner, C., \& Newell, B. R. (2010). In conflict with ourselves? An investigation of heuristic and analytic processes in decision making. Memory \& Cognition, 38, 186-196.

Botvinick, M. M., Cohen, J. D., \& Carter, C. S. (2004). Conflict monitoring and anterior cingulate cortex: An update. Trends in Cognitive Sciences, 12, 539-546.

Damasio, A. R. (1994). Descartes' error: Emotion, reason, and the human brain. New York: Grosset/Putnam.

De Neys, W. (2012). Bias and conflict: A case for logical intuitions. Perspectives on Psychological Science, 7, 28-38. 
De Neys, W., Cromheeke, S., \& Osman, M. (2011). Biased but in doubt: Conflict and decision confidence. PLOS ONE, 6, e15954.

De Neys, W., \& Franssens, S. (2009). Belief inhibition during thinking: Not always winning but at least taking part. Cognition, 113, 45-61.

De Neys, W., \& Glumicic, T. (2008). Conflict monitoring in dual process theories of thinking. Cognition, 106, 1248-1299.

De Neys, W., Moyens, E., \& Vansteenwegen, D. (2010). Feeling we're biased: Autonomic arousal and reasoning conflict. Cognitive, Affective, \& Behavioral Neuroscience, 10, 208-216.

De Neys, W., Vartanian, O., \& Goel, V. (2008). Smarter than we think: When our brains detect that we are biased. Psychological Science, 19, 483-489.

Epstein, S. (1994). Integration of the cognitive and psychodynamic unconscious. American Psychologists, 49, 709-724.

Evans, J. St. B. T. (2003). In two minds: Dual process accounts of reasoning. Trends in Cognitive Sciences, 7, 454-459.

Evans, J. St. B. T. (2008). Dual-processing accounts of reasoning, judgment, and social cognition. Annual Review of Psychology, 59, 255-278.

Evans, J. B. S. T. (2010). Intuition and reasoning: A dual process perspective. Psychological Inquiry, 21, 313-326.

Evans, J. St. B. T., \& Over, D. E. (1996). Rationality and reasoning. Hove, UK: Psychology Press.

Franssens, S., \& De Neys, W. (2009). The effortless nature of conflict detection during thinking. Thinking \& Reasoning, 15, 105-128. 
Gläscher, J., Adolphs, R., Damasio, H., Bechara, A., Rudrauf, D., et al. (2012). Lesion mapping of cognitive control and value-based decision making in the prefrontal cortex. Proceedings of the National Academy of Sciences, doi: 10.1073/pnas.1206608109.

Kahneman, D. (2011). Thinking, fast and slow. New York: Farrar, Strauss, \& Giroux.

Kahneman, D. \& Frederick, S. (2005). A model of heuristic judgement. In K. J. Holyoak \& R. G. Morrison (Eds.), The Cambridge Handbook of Thinking and Reasoning (pp. 267-293). Cambridge, MA: Cambridge University Press.

Kahneman, D., \& Tversky, A. (1973). On the Psychology of Prediction. Psychological Review, 80, 237-251.

Klauer, K. C., \& Singmann, H. (2012). Does logic feel good? Testing for intuitive detection of Logicality in syllogistic reasoning. Journal of Experimental Psychology: Learning, Memory, and Cognition, Online first publication, october 22, doi: 10.1037/a0030530.

Morsanyi, K., \& Handley, S. (2012). Logic feels so good - I like it! Evidence for the intuitive detection of logicality in syllogistic reasoning. Journal of Experimental Psychology: Learning, Memory, \& Cognition, 38, 596-616.

Oppenheimer, D. M. (2008). The secret life of fluency. Trends in Cognitive Sciences, 12, 237-241.

Pennycook, G., Fugelsang, J. A., \& Koehler, D. J. (2012). Are we good at detecting conflict during reasoning? Cognition, 124, 101-106.

Proulx, T., Inzlicht, M., \& Harmon-Jones, E. (2012). Understanding all inconsistency compensation as a palliative response to violated expectations. Trend in Cognitive Sciences, 16, 285-291. 
Stanovich, K. E., \& West, R. F. (2000). Individual differences in reasoning: Implications for the rationality debate? Behavioral and Brain Sciences, 23, 645-726.

Stupple, E. J. N., \& Ball, L. J. (2008). Belief-logic conflict resolution in syllogistic reasoning: Inspection-time evidence for a parallel-process model. Thinking \& Reasoning, 14, 168181.

Thompson, V. A. (2009). Dual process theories: A metacognitive perspective. In J. B. S. T. Evans \& K. Frankish (Eds.), In two minds: Dual processes and beyond. Oxford, UK: Oxford University Press.

Thompson, V. A., \& Morsanyi, K. (2012). Analytic thinking: do you feel like it? Mind and Society, 11, 93-105.

Thompson, V. A., Striemer, C. L., Reikoff, R., Gunter, R. W. \& Campbell, J. I. D. (2003). Syllogistic reasoning time: Disconfirmation disconfirmed. Psychonomic Bulletin \& Review 10, 184189.

Thompson, V. A., Turner, J. A. P., \& Pennycook, G. (2011). Intuition, reason, and metacognition. Cognitive Psychology, 63, 107-140.

Topolinski, S. (2011). A process model of intuition. European Review of Social Psychology, 22, 274-315.

Topolinski, S., \& Strack, F. (2009). The analysis of intuition: Processing fluency and affect in judgments of semantic coherence. Cognition and Emotion, 23, 1465-1503.

Villejoubert, G. (2009). Are representativeness judgments automatic and rapid? The effect of time pressure on the conjunction fallacy. Proceedings of the Annual Meeting of the Cognitive Science society, 30, 2980-2985. 
Wason, P. C., \& Evans, J. (1975). Dual processes in reasoning. Cognition, 141-154. 